\title{
On Cooperative Spectrum Sensing with Improved Energy Detector over Erroneous Control Channel
}

\author{
Narasimha Rao Banavathu and Mohammed Zafar Ali Khan, \\ Department of Electrical Engineering, \\ Indian Institute of Technology - Hyderabad, \\ Kandi, Sangareddy - 502205, Telangana, India \\ Email: \{ee13p1005, zafar\}@iith.ac.in
}

\begin{abstract}
In this paper, we present expressions for optimal number of secondary users (SUs) by minimizing the global error rate for a given fusion rule at the fusion center (FC). Expressions for optimal number of SUs are presented for AND, OR and MAJORITY fusion rules. We show that optimal number of SUs depends on effective probability of false alarm $\left(P_{f e}\right)$ and effective probability of miss detection $\left(P_{m e}\right)$ of a $\mathbf{S U}$ over erroneous control channel. Using improved energy detector as an example feasibility regions are derived for $O R$, AND and MAJORITY rules.

Index Terms-Cognitive radio, cooperative spectrum sensing, improved energy detector, optimization.
\end{abstract}

\section{INTRODUCTION}

Cognitive Radio (CR) [1] is an intelligent wireless communication system that monitors for the presence of active transmission with the aim to achieve efficient utilization of spectrum and reliable communication between primary and secondary users, by changing different modulation strategies and data rates. In many realistic environments due to multipath fading and shadowing, reliability of sensing of SU may deteriorate. In such scenarios cooperation among SUs is needed and reliability in detecting primary user (PU) can be improved by accommodating more SUs [2], [3], [4]. Conventional energy detector for various fading channels is derived in [5]. Cooperative spectrum sensing (CSS) is called $n$-out-of- $K$ voting rule, where FC decides PU is present if at least $n$ out of $K$ SUs report to the FC that identifies $\mathrm{PU}$ is present out of $K$ number of SUs.

One of the practical limitations in CSS is subject to fading and additive noise, the control channel between SU and FC may also experiences errors, which results in poor performance [6]. By allowing most favorable SUs, those whose reporting channel conditions are peak, in the cooperation detection performance can be improved [7]. The benefits of data fusion over decision fusion in presence of imperfect reporting channel is shown in [8]. How ever data fusion requires large bandwidth reporting channel. To make use of limited-bandwidth reporting channel, novel quantization based CSS is proposed in [9], where each SU quantize their observed information and sends it FC over imperfect reporting channel. This scheme performs better than one-bit hard decision combining scheme.

Number of SUs play an important role in describing the performance of CSS. In cooperative CR network, as number of
SUs increase, FC may take long time in deciding the presence of PU because for each time slot only one SU is allowed to send his local decision to the FC [10]. Optimal $n$ and optimal detection threshold over error free control channel is presented using energy detection method. Optimal $n$ over erroneous control channel is presented in [11] and it is shown that AND, OR rule is never optimal to practical cases. Optimal $K$ using OR rule over imperfect control channels for improved energy detector is addressed in [12].

In this paper, we consider optimization of number of SUs over erroneous control channel when AND, OR and MAJORITY rule is used at the FC. We show that AND and MAJORITY rule is optimal for certain values of $K$, $P_{f e}$ and $P_{m e}$. Moreover, as number of SUs increase, it is not guaranteed to improve the performance of cooperative CR network. Therefore, it is important to find the optimal number of SUs to improve the performance. Following [12], we numerically obtain optimal values of detection threshold, $p$ of improved energy detector and optimal $K$ for AND, OR and MAJORITY rule.

This paper is organized as follows. In Section II we present the system model for CSS. In Section III, we obtain the expressions for optimal number of SUs by minimizing the global error rate at FC for AND and MAJORITY rule. We also discuss numerical results with improved energy detector. Finally conclusions are presented in Section IV.

\section{SySTEM MODEL}

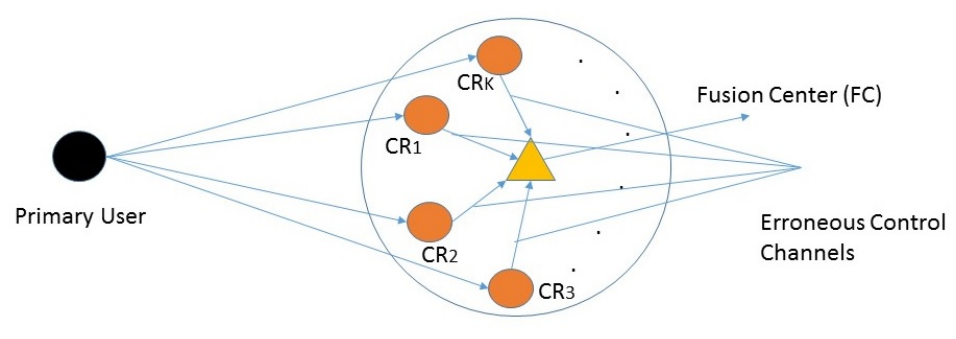

Fig. 1. CSS model considered in this paper.

We consider a cooperative cognitive radio network as shown 
in Fig. 1, where every SU detects activities of the PU independently. Then all $K$ SUs send their local decisions to the FC through erroneous control channels. Let

$H_{1}$ : Indicates PU is present, and

$H_{0}$ : Indicates PU is absent.

The received signal at each secondary user under hypothesis $H_{0}$ and $H_{1}$ is given by

$$
\begin{array}{ll}
x_{i j}=n_{i j}, & H_{0} \\
x_{i j}=s_{i j}+n_{i j}, & H_{1}
\end{array}
$$

where $x_{i j}$ be the $j^{\text {th }}$ sample of the received signal by $i^{\text {th }} \mathrm{SU}$, $s_{i j}$ be the PU signal $j^{t h}$ sample of $i^{t h}$ SU and $n_{i j}$ be the additive white Gaussian noise $N\left(0, \sigma^{2}\right)$. The probability of false alarm, probability of detection and probability of missed detection of $i^{t h} \mathrm{SU}$ with improved energy detector is given by [13]

$$
\begin{aligned}
& P_{f, i}=\operatorname{Pr}\left\{H_{1}^{S U} / H_{0}^{P U}\right\}=\frac{\Gamma\left(b_{0}, \frac{\lambda_{i}}{a_{0}}\right)}{\Gamma\left(b_{0}\right)}, \\
& P_{d, i}=\operatorname{Pr}\left\{H_{1}^{S U} / H_{1}^{P U}\right\}=\frac{\Gamma\left(b_{1}, \frac{\lambda_{i}}{a_{1}}\right)}{\Gamma\left(b_{1}\right)}, \text { and } \\
& P_{m, i}=\operatorname{Pr}\left\{H_{0}^{S U} / H_{1}^{P U}\right\}=1-P_{d, i} .
\end{aligned}
$$

where $a_{0}=\frac{2^{p / 2}}{L} \frac{\sqrt{\pi} \Gamma\left(\frac{2 p+1}{2}\right)-\Gamma^{2}\left(\frac{p+1}{2}\right)}{\Gamma\left(\frac{p+1}{2}\right) \sqrt{\pi}}, a_{1}=\left(1+\gamma_{i}\right)^{p / 2} a_{0}$, $b_{0}=b_{1}=L \frac{\Gamma^{2}\left(\frac{p+1}{2}\right)^{2}}{\Gamma\left(\frac{2 p+1}{2}\right) \sqrt{\pi}-\Gamma^{2}\left(\frac{p+1}{2}\right)}, \Gamma(.,$.$) is the upper incom-$ plete gamma function, $\Gamma($.$) is the gamma function, \lambda_{i}$ is the detection threshold of $i$ th SU, $\gamma_{i}$ is the average signal-to-noise ratio (SNR) of $i$ th SU over $L$ samples and $p$ is the positive power of the improved energy detector.

Following [10], we assume that distance between any two SUs is small, hence each SU experiences identical path loss. Therefore, we can assume that all SUs has same SNR. We also assume that all SUs has same detection thresholds i.e., $\lambda_{1}=$ $\lambda_{2}=\ldots \ldots=\lambda_{K}=\lambda$. Hence they have same probabilities of detection and false alarm. Further more, we assume all control channels between SUs and FC introduce same probability of error, $P_{e}$. In summary

$$
\lambda_{i}=\lambda, P_{f, i}=P_{f}, P_{d, i}=P_{d}, P_{m, i}=P_{m}, P_{e, i}=P_{e} .
$$

Let $P_{d e}$ be the effective probability of detection of a SU over erroneous control channel as seen by the FC and is given by

$$
\begin{aligned}
P_{d e} & =\operatorname{Pr}\left\{H_{1}^{F C} / H_{1}^{P U}\right\}, \\
& =\operatorname{Pr}\left\{H_{1}^{S U} / H_{1}^{P U}\right\}\left\{\times \operatorname{Pr}\left\{H_{1}^{F C} / H_{1}^{S U}\right\}\right. \\
& +\operatorname{Pr}\left\{H_{0}^{S U} / H_{1}^{P U}\right\} \times \operatorname{Pr}\left\{H_{1}^{F C} / H_{0}^{S U}\right\}, \\
& =P_{d}\left(1-P_{e}\right)+\left(1-P_{d}\right) P_{e} .
\end{aligned}
$$

Similarly $P_{f e}$ and $P_{m e}$ be the effective probability of false alarm, effective probability of detection of a SU over erroneous control channel as seen by the FC and is given by

$$
\begin{aligned}
P_{f e} & =P_{f}\left(1-P_{e}\right)+\left(1-P_{f}\right) P_{e}, \\
P_{m e} & =1-P_{d e} .
\end{aligned}
$$

Every SU in CR network makes one bit binary decision (logic 1 indicates PU is present, logic 0 indicates PU is absent) and sends it to the FC over erroneous control channel. At FC all these one bit decisions are fused together to decide the presence or absence of PU.

If FC uses AND rule, then probability of false alarm and probability of miss detection at $\mathrm{FC}$ for a given probability of error in the control channel $\left(P_{e}\right)$, is given by

$$
P_{F, A N D}=P_{f e}{ }^{K}, P_{M, A N D}=1-\left(1-P_{m e}\right)^{K} .
$$

Probability of false alarm and probability of miss detection at FC for OR rule for a given probability of error in the control channel $\left(P_{e}\right)$, is given by

$$
P_{F, O R}=1-\left(1-P_{f e}\right)^{K}, \quad P_{M, O R}=P_{m e}{ }^{K} .
$$

Probability of false alarm and probability of miss detection at FC for MAJORITY rule for a given probability of error in the control channel $\left(P_{e}\right)$, is given by

$$
\begin{aligned}
P_{F, \text { MAJORITY }} & =\sum_{l=\lceil K / 2\rceil}^{K}\left(\begin{array}{c}
K \\
l
\end{array}\right) P_{f e}{ }^{l}\left(1-P_{f e}\right)^{K-l}, \\
& =I_{P_{f e}}\left(\left\lceil\frac{K}{2}\right\rceil, K-\left\lceil\frac{K}{2}\right\rceil+1\right), \\
P_{M, \text { MAJORITY }}=1 & -I_{P_{d e}}\left(\left\lceil\frac{K}{2}\right\rceil, K-\left\lceil\frac{K}{2}\right\rceil+1\right),
\end{aligned}
$$

where $I_{P}(m, n-m+1)=\sum_{i=m}^{n}\left(\begin{array}{c}n \\ i\end{array}\right) P^{i}(1-P)^{n-i}$ is the regularized incomplete beta function with $m, n$ are the positive integers and $0 \leq P<1$.

Global error rate at FC defined as

$$
P_{G E, \chi}=P_{F, \chi}+P_{M, \chi}
$$

where $\chi$ can be one of AND, OR, MAJORITY rules.

\section{Optimization of Number of SeCONDARy Users IN CoOperative SPectrum SENSING OVER ERRONEOUS CONTROL ChanNELS}

In this section we present the expressions for optimal number of SUs for AND, OR and MAJORITY fusion rules by minimizing the global error rate at FC over erroneous control channel.

\section{A. Optimal Number of Secondary Users for AND fusion rule}

Fig. 2 plots the global error rate with respect to detection threshold for five values of $K$ for AND rule at FC. It is observed that cooperation among SUs improves the performance at low values of detection threshold. Performance degrades with $K$ as the detection threshold increases. Clearly, the value of $K$ plays an important role in optimization of CSS. In the following proposition we derive the optimal value of $K$ by minimizing the global error rate of AND rule at FC.

Proposition 1. For the AND fusion rule the optimal SUs, denoted as $K_{A N D}^{*}$, that minimizes the global error rate is given by

$$
K_{A N D}^{*}=\lceil 1 / \beta\rceil, \quad \beta=\frac{\ln \frac{1-P_{m e}}{P_{f e}}}{\ln \frac{1-P_{f e}}{P_{m e}}}
$$






Fig. 2. Global error rate versus detection threshold, for AND rule at FC for $\mathrm{p}=2, \mathrm{~L}=5, P_{e}=0, \mathrm{SNR}=10 \mathrm{~dB}, \mathrm{~K}=[1,5]$, for improved energy detector.

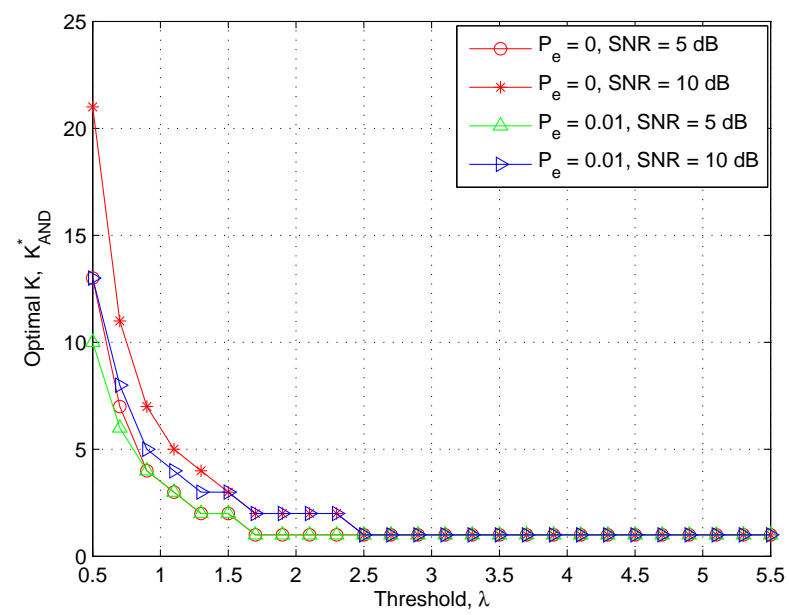

Fig. 3. Effect of control channel errors: Optimal $\mathrm{K}$ versus detection threshold, $\lambda$ for AND rule at FC for $P_{e}=0,0.01, \mathrm{SNR}=5 \mathrm{~dB}, 10 \mathrm{~dB}$, and $p=2$, for improved energy detector.

where $\lceil$.$\rceil indicates ceiling function.$

Proof: Appendix I.

Using proposition 1 , we can make the following remarks which apply to any detector (though the example plots are given for improved energy detector).

Remark 1 (Non Cooperative Scheme). Substituting $K_{A N D}^{*}=$ 1 in (12) and rearranging, we obtain

$$
0<\frac{1}{\beta} \leq 1, \Rightarrow P_{f e} \leq P_{m e} .
$$

Equation (13) implies that cooperation among SUs using AND rule is not beneficial. This is because of increasing the number of SUs will increase the global error rate at FC. For example, if $P_{f e}=P_{m e}$ and $K=2$, from (7) we get $P_{G E, A N D}=2 P_{m e}$.

Remark 2 (Cooperative Scheme). Assume cooperative $C R$ network with $M(M \geq 2)$ SUs are optimal. Substituting $K_{A N D}^{*}=M$ in (12) and rearranging, we get

$$
\begin{aligned}
& M-1<\frac{1}{\beta} \leq M, \Rightarrow P_{m e}\left(1-P_{m e}\right)^{M}>\left(1-P_{f e}\right) P_{f e}{ }^{M}, \\
& \Rightarrow\left(1-P_{f e}\right) P_{f e}{ }^{M-1}>P_{m e}\left(1-P_{m e}\right)^{M-1} .
\end{aligned}
$$

Substituting various values of $M$ in above equation, we get the relation between $P_{f e}$ and $P_{m e}$ where $M$ is optimal. For example, If $M=2$, then

$$
\begin{aligned}
& \quad \Rightarrow\left(1-P_{f e}\right) P_{f e}>\left(1-P_{m e}\right) P_{m e}, \Rightarrow P_{m e}<P_{f e} . \\
& \text { If } M=3 \text {, then } \\
& \Rightarrow\left(1-P_{f e}\right) P_{f e}{ }^{2}>\left(1-P_{m e}\right) P_{m e}{ }^{2}, \Rightarrow P_{m e}<<P_{f e} .
\end{aligned}
$$

In general, $P_{m e}<P_{f e}$. This implies that cooperation among SUs using AND rule is beneficial and there exists an optimal value for number of SUs when $P_{m e}<P_{f e}$. Observe from Fig. 2 that when $\lambda$ is small $\left(P_{m e}<P_{f e}\right)$, as $K$ increases $P_{G E, A N D}$ decreases. But if $\lambda$ is large $\left(P_{m e} \geq P_{f e}\right), P_{G E, A N D}$ increases as $K$ increases.

Fig. 3 plots the optimal $K$ versus detection threshold for AND rule at the $\mathrm{FC}$, for various $P_{e}$ and SNR values. It is observed that for a fixed SNR, optimal number of SUs is decreases as $P_{e}$ increases. This is because errors in control channel reduces the reliability of the decisions received at the FC. It is also observed that for a given $P_{e}$, optimal number of SUs increases as SNR increases.

The above remarks are corroborated from Figs. 2, 3.

Fig. 4 plots the global error rate with respect to detection threshold for five values of $K$, for OR rule at FC. It is observed that cooperation among SUs improves the performance at higher values of detection threshold. Performance degrades with $K$, at the low values of detection threshold. In the following proposition we present the optimal value of $K$ by minimizing the global error rate of OR rule at FC.



Fig. 4. Global error rate versus detection threshold, for OR rule at FC for $\mathrm{p}$ $=2, \mathrm{~L}=5, P_{e}=0, \mathrm{SNR}=10 \mathrm{~dB}, \mathrm{~K}=[1,5]$, for improved energy detector. 


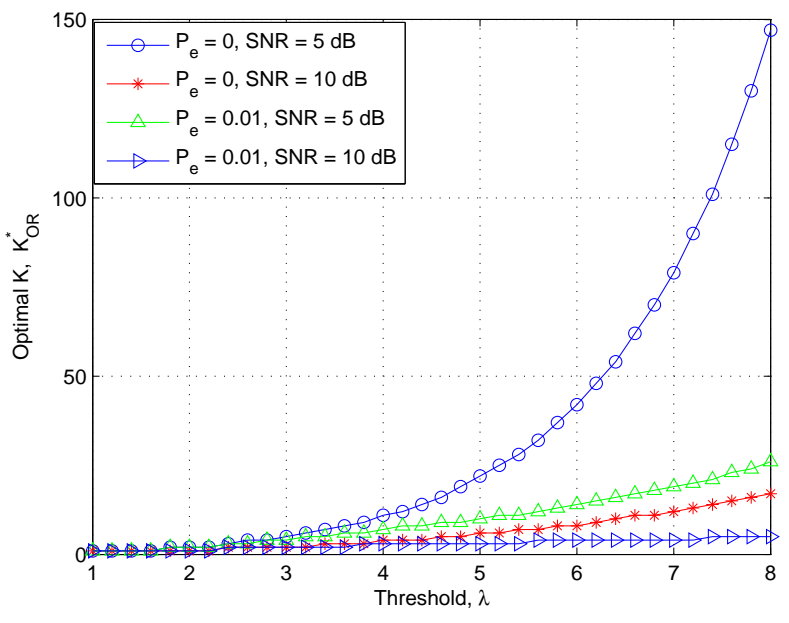

Fig. 5. Effect of control channel errors: Optimal $\mathrm{K}$ versus threshold, $\lambda$ for $\mathrm{OR}$ rule at $\mathrm{FC}$ for $P_{e}=0,0.01, \mathrm{SNR}=5 \mathrm{~dB}, 10 \mathrm{~dB}$, and $p=2$, for improved energy detector.

Proposition 2. In [12, eq. (19)], For the OR fusion rule the optimal number of $S U S$, denoted as $K_{O R}^{*}$, that minimizes the global error rate is given by

$$
K_{O R}^{*}=\lceil\beta\rceil
$$

From proposition 2, we can make the following remarks which apply to any detector (though the example plots are given for improved energy detector) and were not observed in [12].

Remark 3 (Non Cooperative Scheme). Substituting $K_{O R}^{*}=1$ in (14) and rearranging, we obtain

$$
0<\beta \leq 1, \Rightarrow P_{m e} \leq P_{f e} .
$$

Equation (15) implies that cooperation among SUs using OR rule is not beneficial. This is because of increasing the number of SUs will increase the global error rate at FC. For example, if $P_{f e}=P_{m e}$ and $K=2$, from (8) we get $P_{G E, O R}=2 P_{f e}$.

Remark 4 (Cooperative Scheme). Assume Cooperative CR network with $M(M \geq 2)$ SUs is optimal. Substituting $K_{O R}^{*}=$ $M$ in (14) and rearranging, we get

$$
\begin{aligned}
& M-1<\beta \leq M, \Rightarrow\left(1-P_{m e}\right) P_{m e} \leq \leq\left(1-P_{f e}\right)^{M} P_{f e} \\
& \Rightarrow\left(1-P_{f e}\right)^{M-1} P_{f e}<\left(1-P_{m e}\right) P_{m e}^{M-1} .
\end{aligned}
$$

Substituting various values of $M$ in above equation, we get the relation between $P_{f e}$ and $P_{m e}$ where $M$ is optimal, for example if $M=2$, we get $P_{f e}<P_{m e}$ and if $M=3$, we get $P_{f e}<<P_{m e}$

In general, $P_{f e}<P_{m e}$. This means that cooperation among SUs using OR rule is beneficial and there exists an optimal number of SUs in $\mathrm{CR}$ network when $P_{f e}<P_{m e}$. Observe from Fig. 4 that when $\lambda$ is small $\left(P_{f e} \geq P_{m e}\right)$, as $K$ increases $P_{G E, O R}$ increases. But if $\lambda$ is large $\left(P_{f e}<P_{m e}\right), P_{G E, O R}$ decreases as $K$ increases.
Fig. 5 plots the optimal $K$ versus detection threshold for OR rule at the FC, for various $P_{e}$ and SNR values. It is observed that for a given SNR, optimal number of SUs decreases as $P_{e}$ increases. This is because error in control channel reduces the reliability of the decisions received at the FC. It is also observed that for a given $P_{e}$, optimal number of SUs decreases as SNR increases.

The above remarks are corroborated from Figs. 4, 5.

\section{B. Optimal Number of Secondary Users for MAJORITY fusion rule}

Fig. 6 plots the global error rate with respect to detection threshold, for MAJORITY rule at FC. It is observed that for some values of detection threshold, $P_{G E, M A J O R I T Y}$ decreases as $K$ increases. In the following proposition we derive the optimal value of $K$ by minimizing the global error rate of MAJORITY rule at FC.

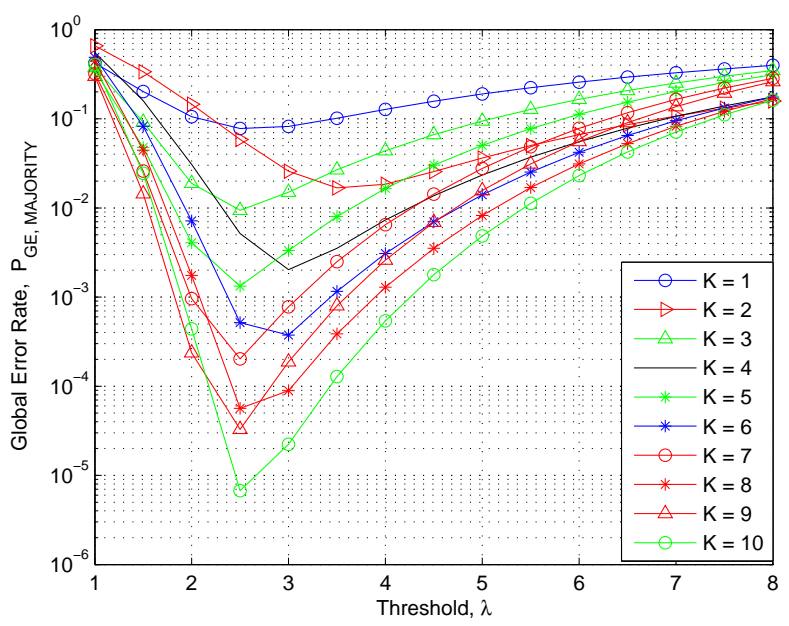

Fig. 6. Global error rate versus detection threshold, for MAJORITY rule at FC for $\mathrm{p}=2, \mathrm{~L}=5, P_{e}=0, \mathrm{SNR}=10 \mathrm{~dB}, \mathrm{~K}=[1,10]$ for improved energy detector.

Proposition 3. For the MAJORITY fusion rule the optimal number of SUs, denoted as $K_{M A J O R I T Y}^{*}$, that minimizes the global error rate is given by

$$
\begin{aligned}
K_{\text {MAJORITY }}^{*} & \approx\left\lceil\frac{2 \beta}{1-\beta}\right\rceil: P_{m e}<P_{f e} \\
& \approx\left\lceil\frac{2}{\beta-1}\right\rceil: P_{m e} \gtrsim P_{f e}
\end{aligned}
$$

where $\beta$ is given by (12)

Proof: Appendix II.

From proposition 3, we can make the following remarks which apply to any detector (though the example plots are given for improved energy detector). We get the relation between $P_{f e}$ and $P_{m e}$ for the following schemes.

Remark 5 (Non Cooperative Scheme). When there is no cooperation among SUs, i.e., $K_{M A J O R I T Y}^{*}=1$, then we consider the following two cases. 
Case A : If $P_{m e}<P_{f e}$, substituting $K_{\text {MAJORITY }}^{*}=1$ in (16) and rearranging, we have

$$
\begin{aligned}
& 0<\frac{2 \beta}{1-\beta} \leq 1, \Rightarrow \beta \geq \frac{1}{3} \\
& \Rightarrow P_{m e}<<P_{f e} .
\end{aligned}
$$

Case B : If $P_{m e} \gtrsim P_{f e}$, substituting $K_{M A J O R I T Y}^{*}=1$ in (17), we have

$$
\begin{aligned}
& 0<\frac{2}{\beta-1} \leq 1, \Rightarrow \beta \geq 3, \\
& \Rightarrow P_{m e}>>P_{f e} .
\end{aligned}
$$

In general $P_{m e}<<P_{f e}$ or $P_{m e}>>P_{f e}$. This implies that when $P_{f e}$ and $P_{m e}$ have different order, cooperation among SUs using MAJORITY rule is not beneficial.

Remark 6 (Cooperative Scheme). Assume Cooperative CR network with $M(M \geq 2)$ SUs is optimal. Substituting $K_{\text {MAJORITY }}^{*}=M$ in (16) and (17), we consider the following two cases.

Case A : If $P_{m e}<P_{f e}$, substituting $K_{\text {MAJORITY }}^{*}=M$ in (16) and rearranging, we have

$$
\Rightarrow M-1<\frac{2 \beta}{1-\beta} \leq M, \Rightarrow \frac{M-1}{M+1} \leq \beta<\frac{M}{M+2},
$$

where $\beta$ is given by (12). Substituting various values of $M$ in above equation, we get the relation between $P_{f e}$ and $P_{m e}$, where $M$ is optimal. When $M$ tends to large value, $P_{f e}$ and $P_{m e}$ have nearly same values and $P_{m e}<P_{f e}$.

Case B : If $P_{m e} \gtrsim P_{f e}$, Substituting $K_{M A J O R I T Y}^{*}=M$ in (17) and rearranging, we have

$$
\Rightarrow M-1<\frac{2}{\beta-1} \leq M, \Rightarrow \frac{M+2}{M} \leq \beta<\frac{M+1}{M-1},
$$

where $\beta$ is given by (12). Substituting various values of $M$ in above equation, we get the relation between $P_{f e}$ and $P_{m e}$, where $M$ is optimal. When $M$ tends to large value, $P_{f e}$ and $P_{m e}$ have nearly same values and $P_{m e} \gtrsim P_{f e}$.

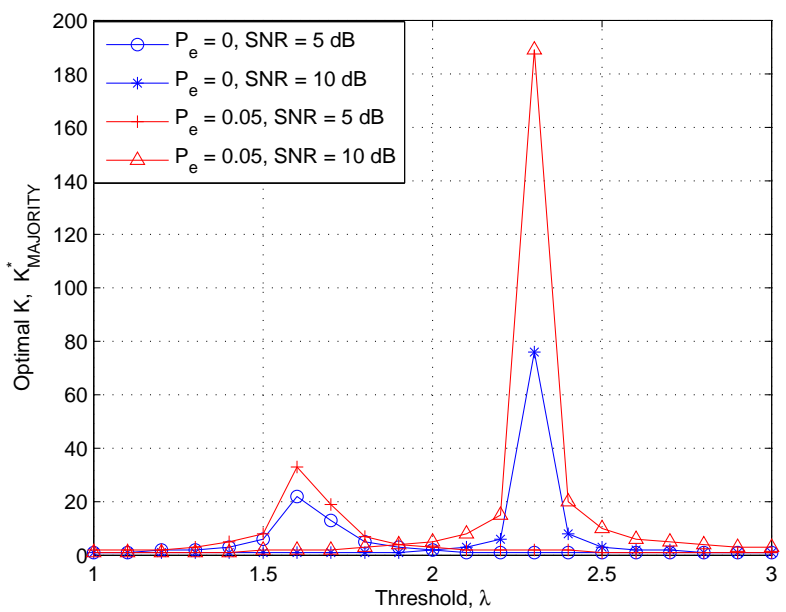

Fig. 7. Effect of control channel errors: Optimal $\mathrm{K}$ versus detection threshold, $\lambda$ for MAJORITY rule at FC for $P_{e}=0,0.05, \mathrm{SNR}=5 \mathrm{~dB}, 10 \mathrm{~dB}$, and $p$ $=2$, for improved energy detector.
In general, $P_{m e}<P_{f e}$ or $P_{m e} \gtrsim P_{f e}$. This means that when $P_{f e}$ and $P_{m e}$ have nearly same values, cooperation among SUs is beneficial and there exists an optimal number of SUs for $P_{m e}<P_{f e}$ or $P_{m e} \gtrsim P_{f e}$. Observe from Fig. 6 that, for some values of detection threshold for which $P_{f e}$ and $P_{m e}$ have nearly same values, $P_{G E, M A J O R I T Y}$ decreases as $K$ increases.

Fig. 7 plots the optimal $K$ versus detection threshold for MAJORITY rule at the FC for various $P_{e}$ and SNR values. It is observed that for a given SNR, optimal number of SUs increases as $P_{e}$ increases. It is also observed that for a given $P_{e}$ in the control channel, optimal number of SUs increases as SNR increases.

The above remarks are corroborated from Figs. 6, 7.

C. Optimization of Improved Energy Detector for AND, OR and MAJORITY fusion Rule

Following [12], we use optimal algorithm, i.e for a given SNR we numerically obtain the values of optimal detection threshold, optimal $p$ of improved energy detector and optimal $K$. Using this optimal algorithm we have plotted the global error rate for AND, OR and MAJORITY rule. We also compare with conventional energy detector $(p=2)$. From Fig. 8 we conclude that performance of MAJORITY rule is better compared to OR and AND rule.

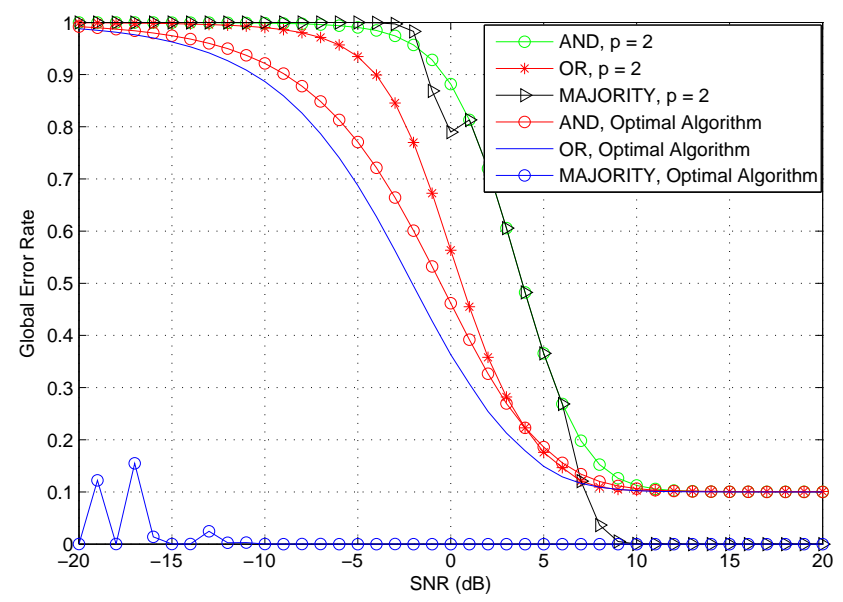

Fig. 8. Global error rate versus SNR, for AND, OR and MAJORITY rule at FC for $P_{e}=0.05, \mathrm{~L}=10$, using optimal $p$, optimal $K$, for improved energy detector.

\section{CONCLUSION}

We have presented the expressions for optimal number of SUs for AND, OR and MAJORITY rule by minimizing the global error rate at FC. It is observed that cooperation among SUs degrades the performance for $P_{f e} \leq P_{m e}$, if FC uses AND rule. On the other hand, if FC uses OR rule, performance of CSS degrades when $P_{m e} \leq P_{f e}$. We discuss the optimality of MAJORITY rule and show that as the number of SUs increase, the global error rate decreases when $P_{f e}$ and $P_{m e}$ have nearly same values. 


\section{APPENDIX I: PROOF OF PROPOSITION 1}

Proof: We define global error rate for AND rule at fusion center as

$$
P_{G E, A N D}=P_{F, A N D}+P_{M, A N D}=f(K)
$$

where $P_{F, A N D}$ and $P_{M, A N D}$ is given by (7).

Optimal number of SUs for AND fusion rule can be obtained by differentiating (18) and equating to 0 , i.e.,

$$
\begin{gathered}
\Delta f(K)=f(K+1)-f(K)=0 \\
\Rightarrow P_{f e}{ }^{K+1}-P_{f e^{K}}-\left(1-P_{m}\right)^{K+1}+\left(1-P_{m}\right)^{K}=0, \\
\Rightarrow 1-\frac{1}{P_{f e}}-\left(\frac{1-P_{m e}}{P_{f e}}\right)^{K+1}+\left(\frac{1-P_{m e}}{P_{f e}}\right)^{K} \frac{1}{P_{f e}}=0 .
\end{gathered}
$$

Simplifying and rearranging, we have

$$
\begin{gathered}
\Rightarrow \frac{1-P_{f e}}{P_{m e}}=\left(\frac{1-P_{m e}}{P_{f e}}\right)^{K}, \\
K_{A N D}^{*}=\lceil 1 / \beta\rceil
\end{gathered}
$$

where $\beta$ is given by (12)

\section{APPENDIX II: Proof OF PROPOSITION 3}

Proof: Global error rate for MAJORITY fusion rule can be expressed as

$$
P_{G E, M A J O R I T Y}=P_{F, M A J O R I T Y}+P_{M, M A J O R I T Y}=g(K)
$$

where $P_{F, M A J O R I T Y}$ and $P_{M, M A J O R I T Y}$ is given by (9) and (10), respectively.

The optimal value of $K$ is obtained when

$$
\Delta P_{G E, M A J O R I T Y}=g(K+1)-g(K)=0
$$

$I_{P_{f e}}\left(\left\lceil\frac{K}{2}\right\rceil, K-\left\lceil\frac{K}{2}\right\rceil+1\right)-I_{P_{f e}}\left(\left\lceil\frac{K+1}{2}\right\rceil, K-\left\lceil\frac{K+1}{2}\right\rceil+2\right)$

$=I_{P_{d e}}\left(\left\lceil\frac{K}{2}\right\rceil, K-\left\lceil\frac{K}{2}\right\rceil+1\right)-I_{P_{d e}}\left(\left\lceil\frac{K+1}{2}\right\rceil, K-\left\lceil\frac{K+1}{2}\right\rceil+2\right)$

Under large $K$, (21) becomes

$$
\begin{aligned}
& I_{P_{f e}}\left(\left\lceil\frac{K}{2}\right\rceil, K-\left\lceil\frac{K}{2}\right\rceil+1\right)-I_{P_{f e}}\left(\left\lceil\frac{K}{2}\right\rceil, K-\left\lceil\frac{K}{2}\right\rceil+2\right) \\
\approx & I_{P_{d e}}\left(\left\lceil\frac{K}{2}\right\rceil, K-\left\lceil\frac{K}{2}\right\rceil+1\right)-I_{P_{d e}}\left(\left\lceil\frac{K}{2}\right\rceil, K-\left\lceil\frac{K}{2}\right\rceil+2\right)
\end{aligned}
$$

Regularized incomplete beta function can also be expressed as follows

$$
I_{P}(m, n)=(1-P)^{n} \sum_{i=m}^{\infty}\left(\begin{array}{c}
n+i-1 \\
i
\end{array}\right) P^{i}
$$

Using (23), we have

$$
I_{P}(m, n+1)-I_{P}(m, n)=\left(\begin{array}{c}
m+n-1 \\
n
\end{array}\right) P^{m}(1-P)^{n}
$$

Simplifying (22) using (24), we get

$$
\left(1-P_{f e}\right)^{K-\left\lceil\frac{K}{2}\right\rceil+1} P_{f e}\left\lceil\frac{K}{2}\right\rceil \approx\left(1-P_{d e}\right)^{K-\left\lceil\frac{K}{2}\right\rceil+1} P_{d e}\left\lceil\frac{K}{2}\right\rceil,
$$

Simplifying and rearranging above equation, we get

$$
\left\lceil\frac{K}{2}\right\rceil \approx \frac{K+1}{\beta+1}, \Rightarrow \frac{K+1}{\beta+1}-1<\frac{K}{2} \lesssim \frac{K+1}{\beta+1}
$$

where $\beta$ is given by (12).

From (25), we get two cases for calculating the optimal choice of $K$.

Case I:

$$
\frac{K+1}{\beta+1}-1<\frac{K}{2}, \Rightarrow K<\frac{2 \beta}{1-\beta} .
$$

Since $P_{f e}+P_{m e}<1, \Rightarrow \beta>0$ and (26) is valid when,

$$
\beta<1, \Rightarrow P_{m e}<P_{f e}
$$

Case II: From (25), we have

$$
\frac{K}{2} \lesssim \frac{K+1}{\beta+1}, \Rightarrow K \lesssim \frac{2}{\beta-1}
$$

(27) is valid when,

$$
\beta \gtrsim 1, \Rightarrow P_{m e} \gtrsim P_{f e}
$$

\section{REFERENCES}

[1] S. Haykin, "Cognitive radio: brain-empowered wireless communications," Selected Areas in Communications, IEEE Journal on, vol. 23 , no. 2, pp. $201-220$, feb. 2005.

[2] A. Ghasemi and E. Sousa, "Collaborative spectrum sensing for opportunistic access in fading environments," in New Frontiers in Dynamic Spectrum Access Networks, 2005. DySPAN 2005. 2005 First IEEE International Symposium on, Nov 2005, pp. 131-136.

[3] B. F. Ian F.Akyildiz and R. Balakrishnan, "Cooperative spectrum sensing in cognitive radio:a survey," vol. 4, no. 4, pp. 40-62, 122010.

[4] S. Atapattu, C. Tellambura, and H. Jiang, "Energy detection based cooperative spectrum sensing in cognitive radio networks," Wireless Communications, IEEE Transactions on, vol. 10, no. 4, pp. 1232-1241, April 2011.

[5] F. Digham, M.-S. Alouini, and M. Simon, "On the energy detection of unknown signals over fading channels," in Communications, 2003. ICC '03. IEEE International Conference on, vol. 5, may 2003, pp. 3575 3579 vol.5.

[6] K. Letaief and W. Zhang, "Cooperative communications for cognitive radio networks," Proceedings of the IEEE, vol. 97, no. 5, pp. 878-893, May 2009.

7] D.-C. Oh and Y.-H. Lee, "Cooperative spectrum sensing with imperfect feedback channel in the cognitive radio systems," Int. J. Commun. Syst., pp. 763-779, May 2010.

[8] S. Chaudhari, J. Lunden, V. Koivunen, and H. Poor, "Cooperative sensing with imperfect reporting channels: Hard decisions or soft decisions?' Signal Processing, IEEE Transactions on, vol. 60, no. 1, pp. 18-28, Jan 2012.

[9] T. T. H. Birkan Yilmaz* and F. Alagoz, "Novel quantization-based spectrum sensing scheme under imperfect reporting channel and false reports," International Journal of Communication Systems, pp. 14591475, Oct 2014.

[10] W. Zhang, R. Mallik, and K. Letaief, "Optimization of cooperative spectrum sensing with energy detection in cognitive radio networks," Wireless Communications, IEEE Transactions on, vol. 8, no. 12, pp. $5761-5766$, december 2009.

[11] N. R. Banavathu and M. Z. A. Khan, "Optimal n-out-of- k voting rule for cooperative spectrum sensing with energy detector over erroneous control channel," in Vehicular Technology Conference (VTC Spring), 2015 IEEE 81st, May 2015, pp. 1-5.

[12] A. Singh, M. Bhatnagar, and R. Mallik, "Optimization of cooperative spectrum sensing with an improved energy detector over imperfect reporting channels," in Vehicular Technology Conference (VTC Fall), 2011 IEEE, Sept 2011, pp. 1-5.

[13] Y. Chen, "Improved energy detector for random signals in gaussian noise," Wireless Communications, IEEE Transactions on, vol. 9, no. 2, pp. 558-563, February 2010. 